\title{
A Retrospective Study into the Use of Prophylactic Antibiotics Pre-Central Venous Catheter Insertion and the Associated Patient Outcomes
}

\section{Joel McCay ${ }^{\star}$ and Jonathan Louden}

Department of Nephrology, James Cook University Hospital, United Kingdom

*Corresponding author: Joel Mccay, Department of Nephrology, James Cook University Hospital, Marton Road, Middlesbrough, North Yorkshire, TS34BW, United Kingdom, Tel: +447894645662, 07894645662; E-mail: joelmccay@hotmail.co.uk

Received date: September 21, 2016; Accepted date: September 26, 2016; Published date: September 29, 2016

Copyright: ( 2016 McCay J, et al. This is an open-access article distributed under the terms of the Creative Commons Attribution License; which permits unrestricted use; distribution; and reproduction in any medium; provided the original author and source are credited.

\begin{abstract}
The risk of bloodstream infection is thought to be as much as 2-3 times more common in patients dialysing from a central venous catheter than those via a fistula. It is not uncommon practice for some nephrology units to use prophylactic antibiotics in the hope of preventing blood stream infections in many patients already with some degree of immunosuppression. As there is no specific guidance we therefore aim to look at the rates of infections in those who received and did not receive antibiotics and their outcomes to try and produce guidelines in relation to their use. 420 lines inserted were analysed over a course of 2 years, the data was analysed and groups divided into those who received antibiotics and those who had not. Those found to be infected were followed up further by analysis of old medical notes and medication prescription charts to see the action taken following a positive line culture and the outcomes associated. In total 97 (23\%) patients didn't receive antibiotics and $323(77 \%)$ did receive prophylactic antibiotics. Positive cultures in the antibiotics and non-antibiotics groups after exclusions of the patient septic prior to line insertion were therefore $9(2.8 \%)$ and $5(5.2 \%)$ respectively. No severe complications from line sepsis occurred in either group and all patients made a full recovery from each group. The risk of developing a catheter related infection leading to a significant event requiring ICU admission or death does not increase regardless of the use of prophylactic antibiotics pre-procedure.
\end{abstract}

Keywords: Access; Infection; Prognosis; CKD

\section{Introduction}

Insertion of Central Venous Catheters (CVC) into femoral or internal jugular veins is a well-established technique performed in patients requiring haemodialysis. CVC insertion acts as another option for the new haemodialysis patient or in transition period between Arteriovenus fistula (AVF) formation for existing End-stage Renal Failure patients. The Procedure is done by clinicians with experience in CVC insertion as some of risks associated are Pneumothorax, Arterial puncture, AVF, nerve injury and failure of procedure [1-4]. 200,000 Lines are inserted annually and the use of USS machine is now recommended by NICE to reduce the complication risk [2]. The risk of bloodstream infection is thought to be as much as 2-3 times more common in patients dialyzing from a central venous catheter than those via a fistula [2,3]. It is not uncommon practice for some nephrology units to use prophylactic antibiotics in the hope of preventing blood stream infections in many patients already with some degree of immune-suppression. The use of antibiotics is not recommended as per NICE guidelines although some nephrology units use teicoplanin and vancomycin in an attempt to reduce gram-positive infections [5]. Teicoplanin is a glycopeptide antibiotic associated with good gram-positive cover and used in South Tees Hospital as prophylaxis. Glycopeptide antibiotics have been shown to reduce incidence of blood stream infections in CVC in certain settings with one such study finding that their use reduced the rates of grampositive infections from $71 \%$ to $20 \%$ [5]. It also concluded the overall incidence of infection in those receiving teicoplanin against those not was $4 \%$ and $24 \%$ respectively [6].
A tremendous effort has been made in recent years across South Tees NHS Trust to improve infection control and it is for this reason that teicoplanin is used prophylactically in the Nephrology unit. Many other units in South Tees using CVC (i.e. Anaesthetics or ICU) do not use prophylactic antibiotics and even within the renal unit antibiotic use varies. As there is no specific guidance we therefore aim to look at the rates of infections in those who received and did not receive antibiotics and their outcomes to try and produce guidelines in relation to their use.

\section{Aims}

A retrospective study at a large DGH teaching hospital to determine the rates of positive line infections in patients who had either received or not received antibiotics before CVC insertion as well as the outcomes to those patients with positive line cultures in the Nephrology unit.

\section{Methods}

420 lines instead were analyzed over a course of 2 years from a documented spreadsheet reserved for audit purposes. It documented the date of insertion, patient number, physician, position, line type, use of monitoring, antibiotic use, success rate and any complications associated. The data was analyzed and groups divided into those who received antibiotics and those who had not. Each patient was analyzed using Sunquest WebIce IT systems to check if there had been any positive blood or line cultures within 1 month since line insertion. Positive cultures were defined as the presence of a pathogen from either the blood obtained from the line site or the line tip itself. Those without any culture results following line insertion were assumed as 
Citation: McCay J, Louden J (2016) A Retrospective Study into the Use of Prophylactic Antibiotics Pre-Central Venous Catheter Insertion and the Associated Patient Outcomes. J Nephrol Ther 6: 260. doi:10.4172/2161-0959.1000260

Page 2 of 3

negative. Those found to be positive were followed up further by analysis of old medical notes and medication prescription charts to see the action taken following a positive line culture and the outcomes associated.

\section{Results}

Of the 420 lines inserted over the 2 year period 171 were inserted by consultants 241 by specialist registers and 8 by other core training doctors. Rates of success were 95\% (401/420) and arterial puncture occurred in 19 patients (5\%). In total 97 (23\%) patients didn't receive antibiotics and 323 (77\%) did receive prophylactic antibiotics. Before in-depth analysis of each patient the number with a positive line culture in the no antibiotics and antibiotics groups were $8(8.2 \%)$ and $19(5.9 \%)$ respectively. Of those with positive line cultures 5 were septic before insertion in the antibiotics group and 3 were in the no antibiotics group and were therefore excluded. A further 4 patients were lost to follow up as notes were unobtainable and so information regarding their outcomes following positive culture was too. Positive cultures in the antibiotics and no-antibiotics groups after exclusions of the patient septic prior to line insertion were therefore $9(2.8 \%)$ and 5 (5.2\%) respectively. Of those, $6(67 \%)$ required no further action following micro-biology or Renal consultant discussion in the antibiotic group and $4(80 \%)$ in the no-antibiotic group. In the noantibiotics group $1(20 \%)$ patient received a course of antibiotics and had their line changed but made a full recovery and was discharged home. In the group who received antibiotics pre-line insertion 3 (33\%) patients required a course of antibiotics and all made a complete recovery (Figure 1).

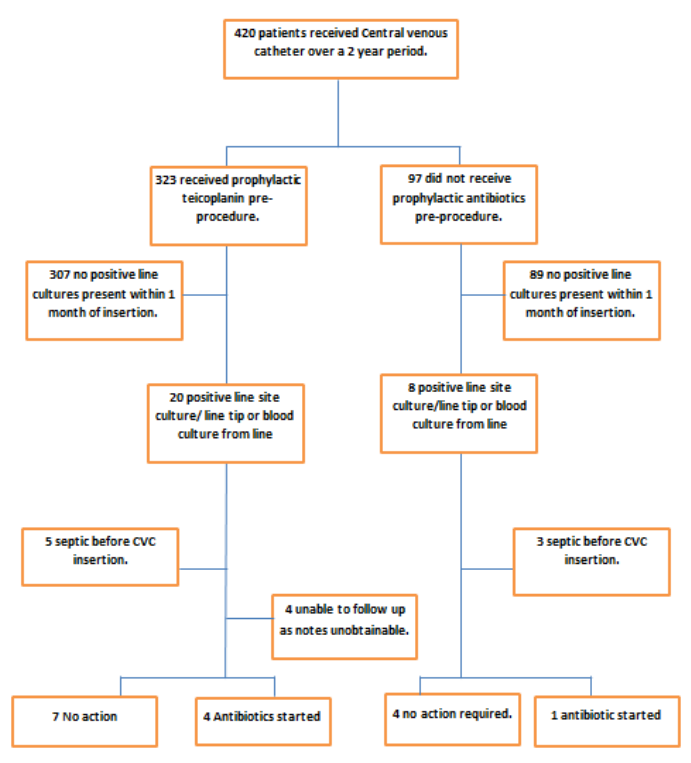

Figure 1: Flow diagram showing the analysis of patients and the outcomes from each group after exclusion and follow up.

Of the patients who did not receive antibiotics there were only 2 isolated organisms, $S$. aureus and Klebsiella pneumoniae (Table 1). In the patients receiving antibiotics the most common organisms isolated were $S$. aureus and Coagulation Negative Staph and a vast array of different organisms as well (Table 2).

\begin{tabular}{|l|l|}
\hline Organism isolated & \\
\hline Staphlococcus aureus & 3 \\
\hline Klebsiella pneumoniae & 2 \\
\hline
\end{tabular}

Table 1: No Antibiotics group.

\begin{tabular}{|l|l|}
\hline Organism isolated & \\
\hline Coagulation Negative Staph & 2 \\
\hline Staph aureus & 2 \\
\hline Staph epidermis & 1 \\
\hline Staph auricularis & 1 \\
\hline Cornyeal bacterium & 1 \\
\hline Mixed enterobacteriaceae & 1 \\
\hline E.coli & 1 \\
\hline Enterobacter cloacae complex & 1 \\
\hline Propionibacterium $s p$ & 1 \\
\hline
\end{tabular}

Table 2: Group who received antibiotics.

\section{Discussion}

From the data analysed above we conclude that outcomes do not worsen regardless of the use of prophylactic teicoplanin. The majorities of people in each group required no action or were commenced on a short course of antibiotics before being discharged home. Current recommendations for line maintenance include review of the site each day as well as changing of dressings every 7 days [7]. Indications for removal of CVC are; a proven organism of catheter related blood stream infection (i.e. S. aureus or Pseudomonas) from the CVC, 2 positive cultures from the same site 48 hours apart and to consider in neutropaenic patients if line sepsis is suspected. This is not however part of the empirical treatment of neutropaenic sepsis [7].

1 of the patients required line removal following advice from microbiology though there were more cases where some of the organisms isolated were indication for line removal. No patients required escalation to level 2 or 3 care as a result of line related infections and no patients died in either group. Off the organisms isolated the split between gram-positive and gram negative was 60:40 in the no antibiotic group and 73:27 in the antibiotic group, suggesting that prophylactic teicoplanin does not provide any added benefit for gram-positive cover.

Prophylactic teicoplanin is not used in many other departments within the hospital and a large meta-analysis in 2002 showed that the use of prophylactic antibiotics does not add any benefit in reducing the risk of blood stream infections [8]. Similar studies have also concluded specifically not to use antibiotics prophylactically and that prevention of blood stream infections is more effective by better surveillance as well as removal of the catheter as soon as it is not needed [9]. A study based in ICU has however shown that prophylactic teicoplain is shown to reduce rates of catheter related infections [6]. Of those who received antibiotics versus those who did not $24 \%$ had a CRI compared to $4.4 \%$ respectively [6]. These results were mostly from temporary CVC as 
Citation: McCay J, Louden J (2016) A Retrospective Study into the Use of Prophylactic Antibiotics Pre-Central Venous Catheter Insertion and the Associated Patient Outcomes. J Nephrol Ther 6: 260. doi:10.4172/2161-0959.1000260

Page 3 of 3

opposed to tunneled lines in renal patients and therefore may not fairly reflect our sample population. More importantly was that the duration of catheterization and catheter site were also independent risk factors for positive line culture. Unfortunately the outcomes of patients with CRI were not performed and are therefore difficult to interpret the significance of this in relation to our results. Other studies have stated that antibiotic locked lines could have benefits in the prevention of line infections and one in particular showed an effective way in reducing blood stream infections are with antibiotic eluting or coated catheters with rates of infection falling from $3.6 \%$ to $1.6 \%$ in the respective groups $[10,11]$.

From a health economics point of view the cost of a $400 \mathrm{mg}$ vile of teicoplanin is $£ 7.32$ as per the BNF and in this short time period of 2 years a total of 323 patients received antibiotics unnecessarily [12]. This in total comes to a total cost of $£ 2364.36$ over the 2 years. This does not take into consideration the cost and time taken to set up the infusion by nursing staff and the need for IV cannulation as well. Though not a substantial figure, in the forever dwindling budget of the NHS it seems an almighty waste of funds for an unnecessary precaution pre-procedure.

\section{Conclusions}

The risk of developing a catheter related infection leading to a significant event requiring ICU admission or death does not increase regardless of the use of prophylactic antibiotics pre-procedure. As a result the use of teicoplanin is wasteful and better surveillance and education of patients on hygiene measures would be a more effective use of healthcare professionals' time and money. Further studies into the duration of catheterisation in renal patients would be of benefit to determine the risks associate with long term tunnel line usage.

\section{Conflict of Interests}

The authors have no conflicts of interest to declare and the results presented in this paper have not been published previously in whole or part anywhere else.

\section{References}

1. Boyce JM (2012) Prevention of central line-associated bloodstream infections in hemodialysis patients. Infect Control Hosp Epidemiol 33: 936-944.

2. National Institute for Health and Care Excellence (2002) Guidance on the use of ultrasound locating devices for placing central venous catheters.

3. Maietta PM (2012) Accidental carotid artery catheterization during attempted central venous catheter placement: a case report. AANA 80: 251-255.

4. Bowdle A (2014) Vascular complications of central venous catheter placement: evidence-based methods for prevention and treatment. Journal of Cardiothoracic and Vascular Anesthesia 28: 358-368.

5. National Institute for Health and Care Excellence (2014) Infection prevention and control.

6. Oncü S, Ozsüt H, Yildirim A, Ay P, Cakar N, et al. (2003) Central venous catheter related infections: Risk factors and the effect of glycopeptide antibiotics. Ann climicrobiol antimicrobiol 2: 3.

7. National Institute for Health and Care Excellence (2012) Neutropenic sepsis: prevention and management in people with cancer.

8. O'Grady NP, Alexander M, Dellinger EP, Gerberding JL, Heard SO, et al. (2002) Guidelines for the prevention of intravascular catheter-related infections. Centers for Disease Control and Prevention. MMWR Recomm Rep 51: 1-29.

9. Shah H, Bosch W, Thompson KM, Hellinger WC (2013) Intravascular catheter-related bloodstream infection. The Neurohospitalist 3: 144-151.

10. Berrington A, Gould FK (2001) Use of antibiotic locks to treat colonized central venous catheters. J Antimicrob Chemother 48: 597-603.

11. Gilbert RE, Mok Q, Dwan K, Harron K, Moitt T, et al. (2016) Impregnated central venous catheters for prevention of bloodstream infection in children (the CATCH trial): a randomised controlled trial. The Lancet 387: 1732-1742.

12. Joint Formulary Committee 2015-2016 (2016) British National Formulary. BMJ Group. 